\title{
Comparative Analysis and Design of Diverse Realizations for FPGA Based Digital PID Controller Using Xilinx SysGen
}

\author{
Hammad Khan, M.Haseeb Akram, Iayan Khalid, Humayun Salahuddin, Abdul Aziz, and Ali Qureshi
}

\begin{abstract}
Digital PID controller is one of the most powerful and efficient controller, which is widely used in industrial control systems. PID controllers can be implemented through either by microprocessors or they can be implemented through FPGA. FPGA based PID controllers are more advantageous in terms of speed and power consumption as compared to software based. Here we design two diverse realizations of FPGA based digital PID controller. One realization is multiplier based which needs multipliers for its implementation and other realization is multiplierless, which is implemented through Distributed Arithmetic Look Up Table (DALUT) method. Distributed arithmetic is an efficient technique to compute inner products using Look Up Tables (LUT). DALUT based PID controller is more efficient because it utilizes less power and hardware resources. Both realizations are simulated in Matlab/Simulink environment. Xilinx SysGen is used to translate both the realizations to bit stream which then can be synthesized, implemented and downloaded to the target FPGA using Xilinx ISE Project Navigator. The results obtained are very helpful for comparative analysis of both the realizations.
\end{abstract}

Index Terms-DALUT, distributed arithmetic, FPGA, look up table, matlab, multiplierless, PID controller, simulink, VHDL, xilinx sysgen .

\section{INTRODUCTION}

PID Controller is one of the most common types of feedback controllers in dynamic systems. Conventionally, we use microcontrollers or microprocessors for implementation of digital PID controller. The memory holds the application programs while the processor fetches, decodes and executes the program instructions. The method has a disadvantage in speed of operation because operation depends on software which has a sequence of instructions and commands which needs many machine cycles to execute[1].

On the other hand FPGA based PID controller are hardware compatible and can be reconfigured according to the requirement which gives advantages in terms of speed and power consumption. But direct implementation of PID controllers on FPGA requires multipliers which requires large number of slices on FPGA. This is the main drawback of multiplier based PID controller [2].

Distributed Arithmetic (DA) architecture, which was first purposed by Peled and Liu in 1974, can be used to

Manuscript received October 12, 2012; revised November 23, 2012

Hammad Khan, M. Haseeb Akram, Iayan Khalid, and Humayun Salahuddin are with the Deptt. of Electronic Engineering, UCET, IUB, Pakistan (e-mail:khammad88@yahoo.com, haseeb_akram88@hotmail.com, iayankhalid@yahoo.com, humayun_s6@yahoo.com)

Abdul Aziz and Ali Qureshi is with the Department of Electronic Engineering, University College of Engineering \& Technology, The Islamia University of Bahawalpur, Pakistan (e-mail: \{abdul.aziz, ali.Qureshi\}@iub.edu.pk) implement multiplierless FPGA based PID controller[3]. The implementation involves look up tables which provides the more flexible and efficient implementation [4].

\section{PID CONTROLLER}

The proportional, integral and derivative control combines to make a PID controller. The design needs more additions or subtractions and multiplications or divisions as complexity increases [5].

Following [6], the general PID equation is given as:

$$
u(t)=K\left(e(t)+\frac{1}{T_{i}} \int^{t} e(t) d t+T_{d} \frac{d e(t)}{d t}\right)
$$

where

$e(t)=$ Error signal

$u(t)=$ Command signal

$\mathrm{K}=$ Gain or Proportional gain

$T_{i}=$ Integration time or rise time

$T_{d}$ = Derivative time

Fig. 1 shows the PID controller used in feedback control system.

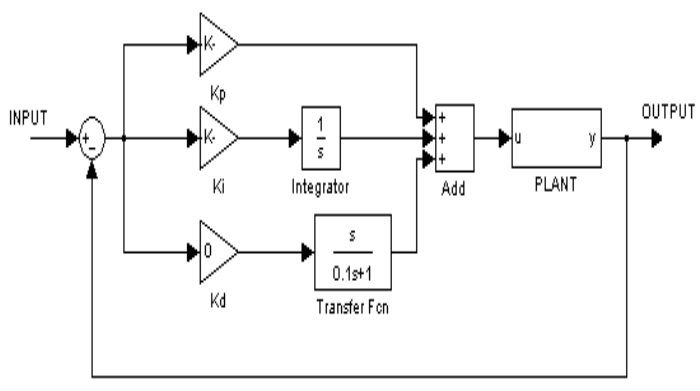

Fig. 1. Feedback control system with PID controller

Taking laplace transform of (1) we get [7]:

$$
U(s)=K\left(E(s)+\frac{1}{s T_{i}} E(s)+s T_{D} E(s)\right)
$$

Now the transfer function of PID controller is:

$$
D(s)=\frac{U(s)}{E(s)}=K\left(1+\frac{1}{s T_{i}}+s T_{D}\right)
$$

Discretized equation (3) we get:

$$
D(z)=K\left(1+\frac{T}{2 T_{i}} \frac{z+1}{z-1}+\frac{T_{D}}{T} \frac{z-1}{z}\right)
$$




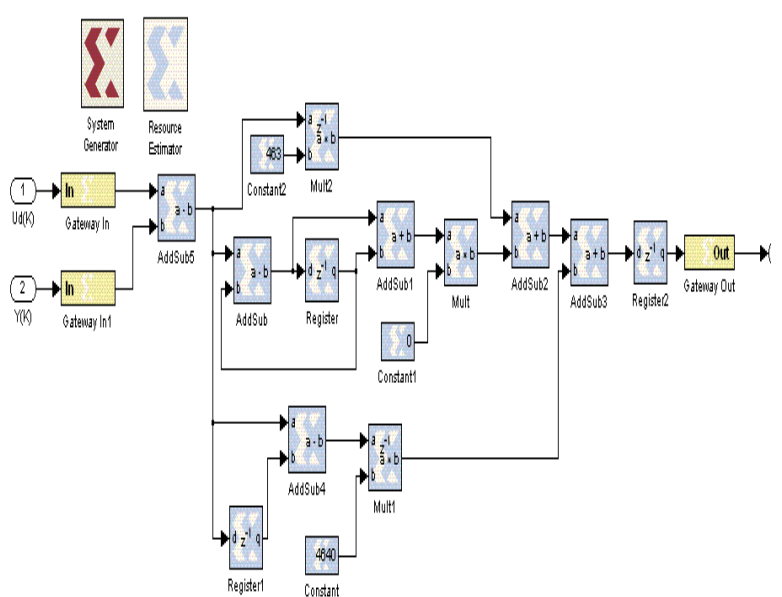

Fig. 2. SysGen implementation of MULTIPLIER based PID controller

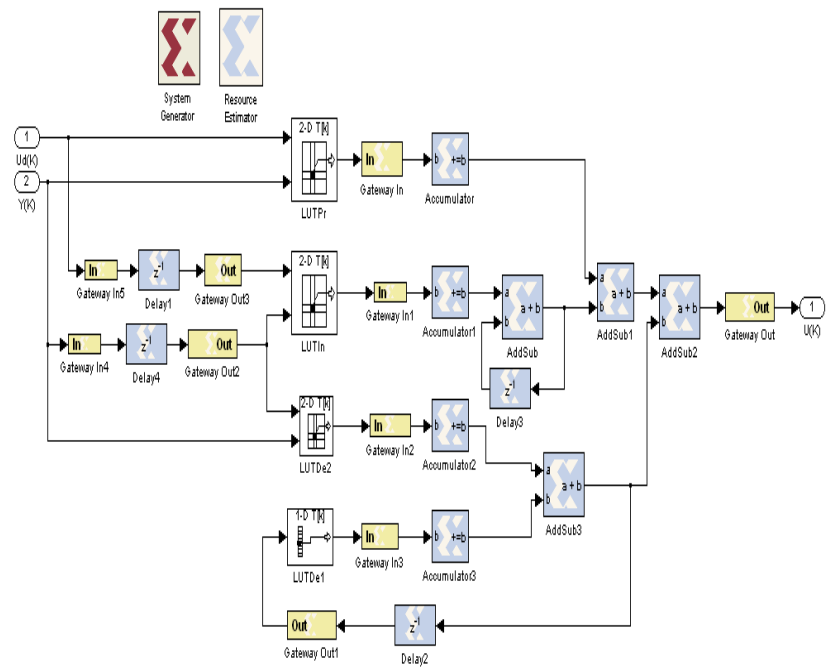

Fig. 3. SysGen implementation of DALUT based PID Controller

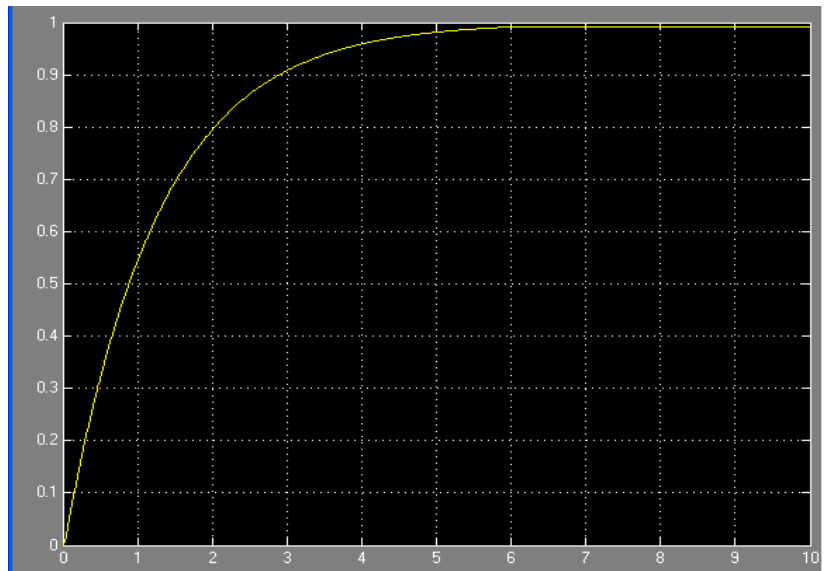

Fig. 4. Step response of multiplier based PID controller

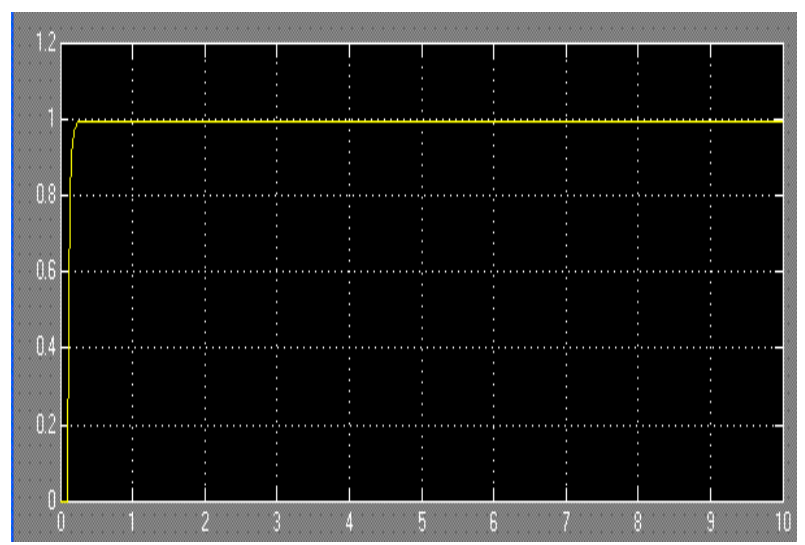

Fig. 5. Step response of DALUT PID controller

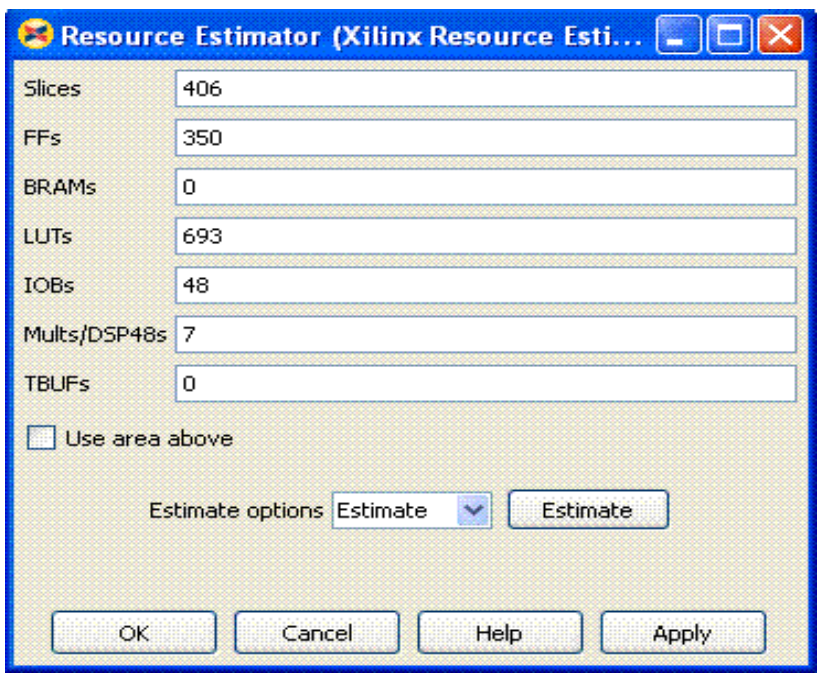

Fig. 6. Synthesis report for Multiplier based PID controller

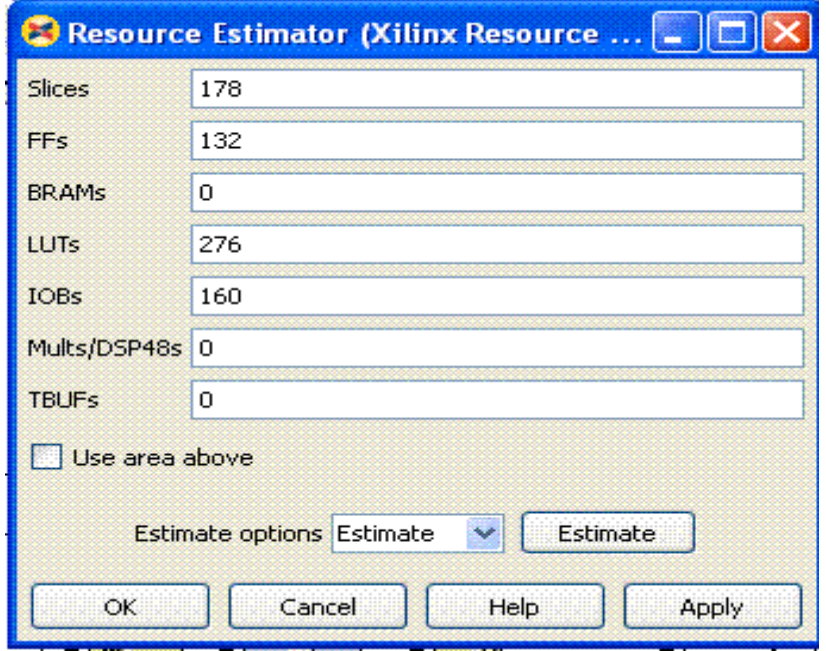

Fig. 7. Synthesis report for DALUT PID controller

\section{A. Distributed Arithmetic (DA)}

Distributed Arithmetic (DA) is a bit-serial algorithm which performs efficient multiplication by using LUT's [8]. Let us consider sum of product (SOP)calculation as:

$$
Y=\sum_{k=0}^{N-1} A_{k} X_{k}
$$

where

$$
\begin{aligned}
& A_{k}=\text { constant coefficient } \\
& x_{k}=\text { input data of size } \mathrm{N} \\
& Y=\text { output }
\end{aligned}
$$

Now assuming that it is a 2's complement fractional number and representing $x_{\mathrm{k}}$ bit-wise format so we get:

$$
x_{k}=-x_{k, 0}+\sum_{j=1}^{M-1} x_{k, j} 2^{-j}
$$

where

$$
\begin{aligned}
& x_{k, j}=\text { jth bit of } x_{k} \\
& x_{k, 0}=\text { Sign Bit } \\
& M=\text { Word Size }
\end{aligned}
$$

Substituting (6) into (5) we get

$$
Y=\sum_{k=0}^{N=1} A_{k}\left[-x_{k, 0}+\sum_{j=1}^{M-1} x_{k, j} 2^{-j}\right]
$$




$$
Y=-\sum_{k=0}^{N-1} A_{k} x_{k, 0}+\sum_{j=1}^{M-1}\left(\sum_{k=0}^{N-1} A_{k} x_{k, j}\right) 2^{-j}
$$

Defining

$$
\begin{gathered}
Z_{j}=\sum_{k=0}^{N-1} A_{k} x_{k, j} \quad(j \neq 0) \\
\text { and } \\
Z_{0}=-\sum_{k=0}^{N-1} A_{k} x_{k, 0}
\end{gathered}
$$

So the output will be written as:

$$
Y=\sum_{j=0}^{M-1} Z_{j} 2^{-j}
$$

In the above equation the SOP of $A_{k}$ 's and $x_{k}{ }^{\prime} s$ is $Z_{j}$ 's . As $x_{k}{ }^{\prime} s$ may be 0 or 1 and $A_{k}$ 's are constant coefficients so there are $2^{N}$ possible values of $Z_{j}$.

These values can be pre-calculated and stored in Look Up Tables(LUT's) or ROM. The address of the values of $x_{k}$ 's are based on $K=0 \rightarrow N-1$. The output can be calculated by shifting and addition operations for all $Z_{j}$ 's from jth bit.

\section{B. DA based PID Controller}

The hardware optimization is the key step involved in implementation of PID on FPGA. DA based PID controller is area efficient as it can fit on very small FPGA chips. This will reduce the cost and improve the efficiency of system [9].

Let us consider equation (1) in which we cannot implement derivative control purely because it will amplify noise. So its gain must be limited. So in this way noise can be reduced. As in [10], approximating the $s T_{D}$ in equation (2) as follows:

$$
s T_{D}=\frac{s T_{D}}{1+s T_{D} / N}
$$

This approximation is correct for the low frequencies while at high frequencies the gain is limited by $N$ ranges from 3 to 20 . While working with analog controller we have to limit the derivative controller gain. Later it was found that only a fraction ' $f$ ' of the command signal act on the proportional gain.

Now improved PID algorithm is given by:

$$
U(s)=K\left(f U_{d}(s)-Y(s)+\frac{1}{s T_{i}}\left(U_{d}(s)-Y(s)\right)-\frac{s T_{D}}{1+s T_{D} / N} Y(s)\right)
$$

Now discretized the above equation we get:

$$
U(K)=P R(K)+I N(K)+D E(K)
$$

where

$$
\begin{gathered}
P R(K)=K\left(f U_{d}(K)-Y(K)\right) \\
I N(K)=I N(K-1)+\frac{K}{T_{i}}\left(U_{d}(K-1)-Y(K-1)\right) \\
D E(K)=\frac{T_{d}}{T_{d}+N T} D E(K-1)-\frac{K T_{d} N}{T_{d}+N T}(Y(k)-Y(K-1)
\end{gathered}
$$

Now by using the DA algorithm the above equations are the form of:

$$
\begin{gathered}
P R(K)=\sum_{j=0}^{M-1}\left(K f \times U_{d}(K)[j]-K \times Y(K)[j]\right) \times 2^{j} \\
I N(K)=I N(K-1)+\sum_{j=0}^{M-1} \frac{K T}{T_{i}}\left(U_{d}(K-1)[j]-Y(K-1)[j]\right) \times 2^{j} \\
D E(K)=\sum_{j=0}^{M-1}\left(\frac{T_{d}}{T_{d}+N T} D E(K-1)[j]-\frac{K T_{d} N}{T_{d}+N T}(Y(k)[j]-Y(K-1)[j])\right) \times 2^{j}
\end{gathered}
$$

where $\mathrm{T}$ is the sampling time.

The results of $\left(K f \times U_{d}(K)[j]-K \times Y(K)[j]\right)$,

$$
\begin{aligned}
& \left(\frac{K T}{T_{i}}\left(U_{d}(K-1)[j]-Y(K-1)[j]\right)\right),\left(\frac{T_{d}}{T_{d}+N T} D E(K-1)[j]\right) \\
& \text { And }\left(-\frac{K T_{d} N}{T_{d}+N T}((Y(k)[j]-Y(K-1)[j]))\right.
\end{aligned}
$$

Can be pre-calculated and stored in respective LUT's denoted as $L U T_{\mathrm{Pr}}, L U T_{I n}, L U T_{D e 1}$ and $L U T_{D e 2}$ respectively.

The contents of these LUT's are:

TABLE I: CONTENTS OF $L U T_{\mathrm{Pr}}$

\begin{tabular}{|c|c|c|}
\hline$U_{d}(K)[j]$ & $Y(K)[j]$ & $L U T_{\operatorname{Pr}}$ \\
\hline 0 & 0 & 0 \\
\hline 0 & 1 & $-K$ \\
\hline 1 & 0 & $K f$ \\
\hline 1 & 1 & $K f-K$ \\
\hline
\end{tabular}

\begin{tabular}{|c|c|c|} 
TABLE II: CONTENTS OF $L U T_{\text {In }}$ \\
\begin{tabular}{|c|c|c|}
\hline$U_{d}(K-1)[j]$ & $Y(K-1)[j]$ & $L U T_{\text {In }}$ \\
\hline 0 & 0 & 0 \\
\hline 0 & 1 & $-K T / T_{i}$ \\
\hline 1 & 0 & $K T / T_{i}$ \\
\hline 1 & 1 & 0 \\
\hline
\end{tabular}
\end{tabular}

TABLE III: CONTENT OF $L U T_{D e 2}$

\begin{tabular}{|c|c|c|}
\hline$Y(K)[j]$ & $Y(K-1)[j]$ & $L U T_{D e 2}$ \\
\hline 0 & 0 & 0 \\
\hline 0 & 1 & $\frac{K T_{d} N}{T_{d}+N T}$ \\
\hline 1 & 0 & $-\frac{K T_{d} N}{T_{d}+N T}$ \\
\hline 1 & 1 & 0 \\
\hline
\end{tabular}

TABLE IV: CONTENT OF $L U T_{D e 1}$

\begin{tabular}{|c|c|}
\hline$D E(K-1)$ & $L U T_{D e 1}$ \\
\hline 0 & 0 \\
\hline 1 & $\frac{T_{d}}{T_{d}+N T}$ \\
\hline
\end{tabular}

\section{IMPLEMENTATION AND RESULTS}

SysGen implementation of Multiplier based PID controller and DALUT based PID Controller for speed control of a DC motor are shown in Fig. 2 and 3 respectively. Step response of the two realizations are shown in figure 4 and 5 . These responses clearly shows that the time response 
of DALUT based PID Controller is much better because it has small rise time as compared to Multiplier based PID controller.

Fig. 6 and 7 shows the comparison of synthesis report for both PID controllers. The synthesis report is generated using Xilinx ISE tool for Spartan 3E [11],[12] . It provides the information about slices, Flip-Flops, Input Output blocks, LUT's and Multipliers. We can see that Multiplier based PID controller requires large number of hardware resources as compared to DALUT based PID controller. DALUT based controller requires 178 Slices as compared to 406 slices and 132 Flip Flops as compared to 350.

\section{CONCLUSION}

FPGA based PID controllers are more advantageous than conventional microprocessor based PID controllers due to high speed and low power consumption. Two realizations of FPGA based PID controller are considered, one is multiplier based and other is DALUT based. Implementation of Multiplier based PID controller requires large number of hardware resources, power consumption and poor step response whereas DALUT based PID controller is a compact design with optimal utilization of hardware resources, less power consumption and a good quality step response. DALUT based PID controller reduces the cost of FPGA design because Look Up Tables are utilized to improve performance of multiplication operation. DALUT based implementation utilizes only 43\% hardware resources as compared to Multiplier based PID controller. Due to flexibility of Look Up Tables DALUT based PID controller can be extended to accommodate adaptive algorithm.

\section{ACKNOWLEDGMENT}

We would like to thank our respected teachers and parents for their guidance and ever present support.

We are also very much thankful to Dr. Muhammad Mukhtar, the Vice Chancellor of the university for his efforts to facilitate and provide us most suitable environment for research and development.

\section{REFERENCES}

[1] V. Gupta, K. Khare, and R. P. Singh, "Efficient FPGA Design and Implementation of Digital Controllers in Simulink," International Journal of Recent Trends in Engineering, vol. 2, no. 6, Nov 2009.

[2] I. Grout, “ Digital System Design with FPGAs and CPLDs,” 2008 Edition, Elsevier Newnes Press Publication, pp 661-674

[3] A Peled and B. Liu, "A New Hardware Realization of Digital Filters," IEEE Trans. ASSP, ASSP-22, pp. 456-462, Dec1974.

[4] A. Cataldo, "Low-Priced FPGA options set to expand," Electronic Engineering Times Journal, no. 1361, pp. 38-65, USA 2005.

[5] G. F. Franklin, J. D. Powell, and M. L. Workman, "Digital Control of Dynamic Systems, "Addison -Wesley Publishing Company, 1990.

[6] B. Wittenmark, K. J Astrom, and K. E. Arzen, "Computer Control: An Overview," Technical Report, Department of Automatic Control, Lund Institute of Technolgy, Sweden, April 2003.

[7] V. Gupta, K. Khare, and R. P. Singh, "Efficient Design and FPGA Implementation of Digital Controllers in Xilinx SysGen," International Journal of Electronics Engineering, vol. 2, no. 1, 2010, pp. 99-102.

[8] A. White, "Application of distributed Arithmetic to Digital Signal Procesing : A Tutorial Review,” IEEE Accoustic, Speech and Signal Processing Magazine, vol. 6, pp. 4-19, 1989.

[9] S. Chivapreech, S. Yimman, C. Pradabpet, and K. Dejhan, "FPGAbased multiplier Less Digital PID Controller Using Distributed Arithmetic," ACLAR'05 international conference, Bangkok, Thailand.

[10] Y. F. Chan, M. Moallem, and W. Wang, "Design and Implementation of Modular FPGA-Based PID Controllers," IEEE Trans. on Industrial Electronics, vol. 54, no. 4, pp. 1898-1906, August 2007

[11] Spartan 3E Data sheet. [Online]. Available: http://www.xilinx.com.

[12] Diligent Spartan-3 System Board. [Online]. Available: http://www.digilentinc.com/Data/Products/S3BOARD/S3BOARDbrochure.pdf.

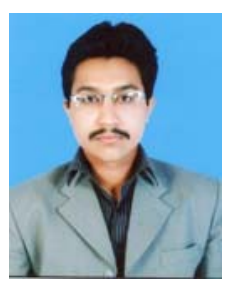

Hammad Khan was born in Bahawalpur on $2^{\text {nd }}$ of June, 1988. Earn Engineering degree with specialization in Electronics from Islamia University Bahawalpur. Major field of study includes Control Systems, Hybrid Electrical Vehicles, Audio Signal processing and Engineering management. He has worked as Technical Valuation Manager In Abl, Pakistan. He is also the reviewer and TPC member of a number of international conferences including $3^{\text {rd }}$ ICCTD 2011 China, IEEE PECON 2012, SHUSER 2012, IEEE ISIEA 2012 and ISWTA 2012(Malaysia) Mr. Hammad Khan is also the member of GARP and Session Chair of "Smart Systems and Softwares" in WICT 2012. 